\title{
Does thrombectomy inhibit effect of ischaemic postconditioning in STEMI? True or not?
}

\author{
Cheol Woong Yu
}

The acute restoration of blood flow to the ischaemic myocardium during reperfusion therapy for ST-segment elevation myocardial infarction (STEMI) may induce further injuries to the myocardium. This phenomenon, reperfusion injury, can paradoxically reduce the beneficial effects of myocardial reperfusion. Studies using various animal models have reported that the reperfusion injury can account for up to $50 \%$ of the total size of myocardial infarction. ${ }^{1}$ As such, minimising reperfusion injury could have significant clinical implications. In clinical practice, the mortality rate for STEMI has reached a plateau with a 1-year mortality of $10 \%$, with approximately $25 \%$ patients developing heart failure, despite an early and successful reperfusion. ${ }^{12}$ Thus, there is a need for further improvements in the treatment of STEMI. The prevention of reperfusion injury may help to improve outcome following the most widely applied form of reperfusion therapy, primary percutaneous coronary intervention (PCI).

There are four categories of reperfusion injury in STEMI: stunning, reperfusion arrhythmia, no reflows/slow reflow by a microvascular obstruction (MVO) and lethal reperfusion injury. ${ }^{1}$ Different types of reperfusion injury may occur at the same time and ultimately determine the infarct size. ${ }^{13}$ Different methods have been examined to prevent reperfusion injury (figure 1). ${ }^{13}$ No reflow/slow reflow and lethal reperfusion injury often occur simultaneously. These two types of reperfusion injury can result in irreversible myocardial necrosis. ${ }^{1}$ It is unknown which one of the two reperfusion injuries contribute more to the final myocardial infarct size. It is suspected that the contribution of each reperfusion injury to final infarct size might depend on the nature of the lesions and is expressed roughly by the sum of the two types of reperfusion injury in clinical practice. Efforts to reduce these two reperfusion

Cardiology, Korea University Anam Hospital, Seoul, Republic of Korea

Correspondence to Dr Cheol Woong Yu, Cardiology, Korea University Anam Hospital, Seoul 02841, Republic of Korea; ycw717@naver.com injuries have focused on thrombectomy, ischaemic postconditioning (IPC) and pharmacological conditioning and other such strategies. Recently, deferred stenting, defined as delaying of stent implantation until thrombus regressed after reperfusion with minimalistic approach, has been emphasised as another method to reduce no reflow. ${ }^{4}$

Myocardial stunning, manifested as myocardial dysfunction, is reversible within several days to weeks. Reperfusion arrhythmia can be prevented and has little impact on the infarct size. ${ }^{1}$ No reflow following a reperfusion therapy is caused by MVO, the mechanism of which is the distal embolisation of the plaque clots. Other possible mechanisms include vasospasm, extrinsic compression of the vessel by tissue oedema, in situ thrombosis and other such mechanisms. MVO, resulting in myocardial tissue hypoperfusion, can manifest angiographically as normal reflow, slow reflow or no reflow, with the most severe form being no reflow. MVO increases the infarct size, and the larger the MVO size, the worse is the

\section{Prevention of Reperfusion Injury in STEMI}

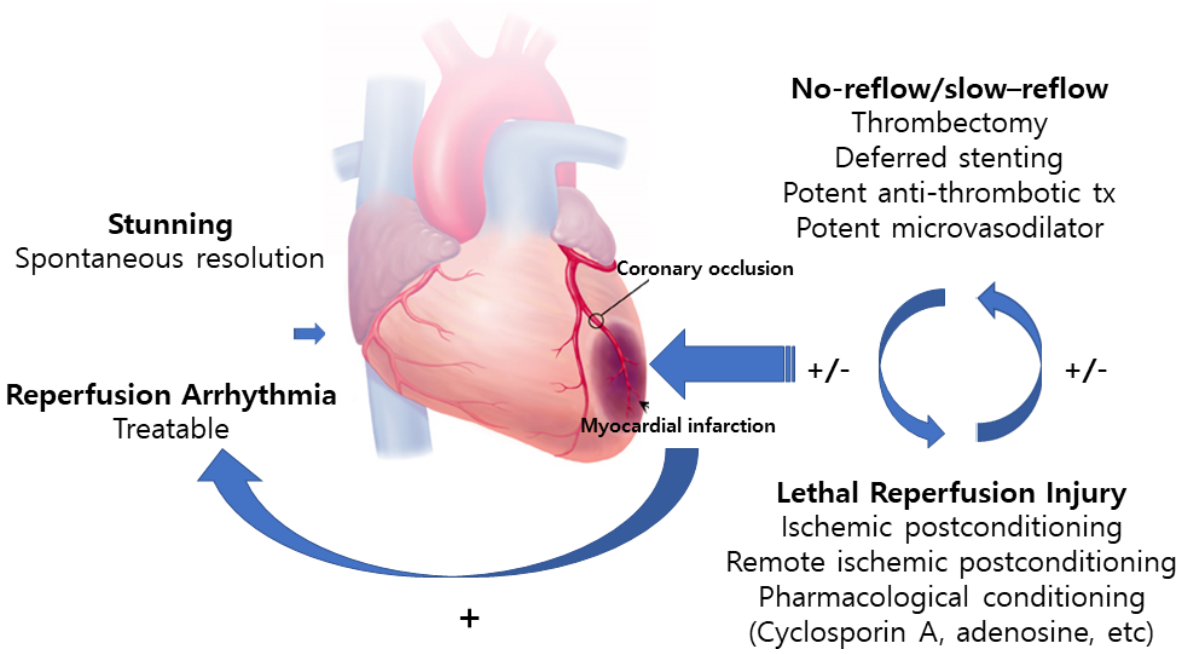

Figure 1 Reperfusion injury during primary percutaneous coronary intervention and methods to prevent reperfusion injury in ST-segment elevation myocardial infarction (STEMI). No reflow/ slow reflow and lethal reperfusion injury can cause irreversible myocardial damage independently of each other and a summation of these two injuries effect a final infarction size. Different interventions to reduce reperfusion injury may mimic or interfere with other intervention. Larger infarct size promotes reperfusion ventricular arrhythmia. 
disappointing results of IPC. ${ }^{3}$ Why are there the difference of IPC between experimental studies and clinical studies? There are several reasons as follows. In experimental studies, the STEMI model was developed by the ligation of a nearly normal artery of young animals, and the ischaemic time was as short as $90 \mathrm{~min} .{ }^{13}$ However, in clinical trials, the coronary artery lesions of patients were highly advanced arteriosclerotic lesions with large thrombus burden and frequently associated with longer ischaemic time and various underlying comorbidities. ${ }^{3} 7$ These differences may mainly contribute to the lack of efficacy of IPC in clinical studies, although IPC reduced the size of myocardial infarction in animal studies.

Another reason may be an interaction between different interventions to reduce reperfusion injuries. Different interventional therapies to prevent reperfusion injury may mimic or interfere with the effects of other interventions. ${ }^{3}$ For example, thrombectomy may partly mimic IPC through the progressive restoration of blood flow during thrombus extraction, but it may paradoxically promote distal embolisation of the thrombus to exert a harmful effect. ${ }^{36}$ As the authors suggested, it is possible that thrombectomy delay starting of IPC during reperfusion and thus interfere with its effectiveness. IPC performed by repeated ballooning within a target lesion may lead to an increase in MVO by promoting the distal embolisation and exacerbating reperfusion injury by no reflow. ${ }^{3}$ Deferred stenting may also mimic the effect of IPC by the gradual restoration of the blood flow. Therefore, when two or more procedures are used simultaneously in one lesion, it may be difficult to accurately evaluate the effect of one procedure.

DANAMI-3-iPOST, designed to determine whether IPC can improve the clininical outcomes in patients with STEMI, did not prove the effect of IPC. ${ }^{8}$ However, thrombectomy was performed in $57.8 \%$ of all patients in this study. ${ }^{8}$ The authors assumed that the interaction between thrombectomy and IPC might affect the outcomes. Thus, they further stratified the patients according to the use of thrombectomy in the study. During a median 35-month follow-up period, there was a significant interaction between IPC and thrombectomy on a composite outcome of all-cause mortality and hospital admissions for heart failure $(p=0.004)$. IPC with primary PCI reduced the risk of all-cause mortality and hospitalisations for heart failure compared with conventional PCI in patients not treated with thrombectomy (10\% vs $18 \%$, adjusted HR 0.55, $\mathrm{p}=0.016)$. In patients treated with thrombectomy, there was no significant difference between ischaemic postconditioning and conventional PCI (adjusted HR 1.18, $\mathrm{p}=0.62$ ).

Based on the graphs presented by the authors, among the four patient groups, the group treated with only conventional PCI showed the highest incidence of the primary endpoints, mainly driven by allcause mortality. Moreover, the event rates in the remaining three groups (two groups with thrombectomy and one group undergoing IPC without thrombectomy) were similar.

It is difficult to interpret this result. The authors insisted that IPC did not appear to be effective in patients treated with thrombectomy because thrombectomy interfered with the efficacy of IPC. However, how should we explain the similar event rates between the patients treated with thrombectomy and patients undergoing IPC without thrombectomy?

As described above, a partial effect of IPC could be achieved with thrombectomy by slowing the reperfusion of the bloodstream. ${ }^{4}$ Therefore, we could understand that implementing IPC through repetitive ballooning could not provide an additional effect. Theoretically, thrombectomy might reduce reperfusion injury by simultaneously reducing distal embolisation and achieving partial IPC effect. This mechanism could explain why there was no difference in the event rates between the IPC and the conventional PCI group in patients with thrombectomy and why the event rate was lower than that in patients with only conventional PCI, at the same time.

Differentiation of the two scenarios is very important in practice. If the two treatments merely interfere with each other, we should conclude that all patients with STEMI should avoid the combined use of IPC and thrombectomy. However, if one treatment shares the effects of another one, the choice of treatment should be done according to differential risk of reperfusion injuries evaluated based on the characteristics of the lesions in practice.

In this study, thrombectomy was performed at the discretion of the operators; therefore, it was likely that thrombectomy was frequently performed for lesions with a large thrombus burden. However, the authors did not analyse the thrombus burden in each lesion. Therefore, it is reasonable to speculate that patients treated with thrombectomy were likely to have more thrombotic lesions and a higher risk of myocardial damages due to no reflow/slow reflow rather than a lethal reperfusion injury. In these patients, thrombectomy was likely to be effective in reducing reperfusion injury by simultaneously reducing distal embolisation and the partial IPC effect. Conversely, patients without thrombectomy were likely to have less thrombotic lesions and a higher risk of myocardial damages due to lethal reperfusion injury rather than no reflow/slow flow. Therefore, IPC was likely to be effective. If this assumption would have been correct, the authors have chosen the optimal treatment, whether intended or unintentional, except for patients who did not received any treatment to prevent reperfusion injury.

The present Heart study by NepperChristensen et $a l^{9}$ is a clinical study to evaluate the interactions between thrombectomy and IPC performed to reduce reperfusion injury. Regardless of the reliability of the proposed mechanism, the present study is valuable in that it provided inspiration for future research. Efforts to reduce reperfusion injury in patients with STEMI should be attempted, and they may be effective if an appropriate treatment strategy is selected. Future research should focus on the selection of an optimal treatment based on lesion characteristics to reduce reperfusion injury in patients with STEMI.

Acknowledgements The author thank Dr H D Kook and Dr H J Lee.

Funding The authors have not declared a specific grant for this research from any funding agency in the public, commercial or not-for-profit sectors.

Competing interests None declared.

Patient consent for publication Not required.

Provenance and peer review Commissioned; internally peer reviewed.

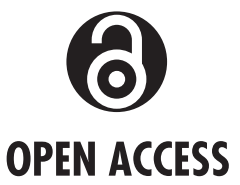

Open access This is an open access article distributed in accordance with the Creative Commons Attribution Non Commercial (CC BY-NC 4.0) license, which permits others to distribute, remix, adapt, build upon this work non-commercially, and license their derivative works on different terms, provided the original work is properly cited, appropriate credit is given, any changes made indicated, and the use is non-commercial. See: http:/l creativecommons.org/licenses/by-nc/4.0/.

(c) Author(s) (or their employer(s)) 2020. Re-use permitted under CC BY-NC. No commercial re-use. See rights and permissions. Published by BMJ.

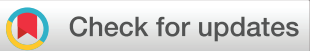

To cite Yu CW. Heart 2020;106:3-5.

Published Online First 14 October 2019

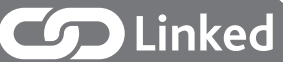

- http://dx.doi.org/10.1136/heartjnl-2019-314952 
Heart 2020;106:3-5.

doi:10.1136/heartjnl-2019-315389

ORCID iD

Cheol Woong Yu http://orcid.org/0000-0002-58714562

\section{REFERENCES}

1 Yellon DM, Hausenloy DJ, Derek M. Myocardial reperfusion injury. N Eng/ I Med 2007;357:1121-35.

2 Terkelsen CJ, Sørensen JT, Maeng M, et al. System delay and mortality among patients with STEMI treated with primary percutaneous coronary intervention. JAMA 2010;304:763-71.
3 Lønborg JT. Targeting reperfusion injury in the era of primary percutaneous coronary intervention: hope or hype? Heart 2015;101:1612-8.

4 Mahmoud AN, Saad M, Elgendy AY, et al. Deferred or immediate stent implantation for primary percutaneous coronary intervention: a meta-analysis of randomized trials. Catheter Cardiovasc Interv 2018;91:260-4.

5 Eitel I, de Waha S, Wöhrle J, et al. Comprehensive prognosis assessment by CMR imaging after STsegment elevation myocardial infarction. J Am Coll Cardiol 2014;64:1217-26.

6 Jolly SS, James S, Džavík V, et al. Thrombus aspiration in ST-segment-elevation myocardial infarction: an individual patient meta-analysis: thrombectomy Trialists collaboration. Circulation 2017;135:143-52.
7 Freixa X, Bellera N, Ortiz-Pérez JT, et al. Ischaemic postconditioning revisited: lack of effects on infarct size following primary percutaneous coronary intervention. Eur Heart $J$ 2012:33:103-12.

8 Engstrøm T, Kelbæk H, Helqvist S, et al. Effect of ischemic postconditioning during primary percutaneous coronary intervention for patients with ST-segment elevation myocardial infarction: a randomized clinical trial. JAMA Cardiol 2017;2:490-7.

9 Nepper-Christensen L, Høfsten DE, Helqvist S, et al. Interaction of ischaemic postconditioning and thrombectomy in patients with ST-elevation myocardial infarction. Heart 2020;106:24-32. 